\title{
The sensing of retinal motion
}

\author{
WALTER C. GOGEL \\ University of California, Santa Barbara, California 93106
}

\begin{abstract}
The apparent relative motion of physically stationary objects that frequently occurs as the head is moved in a frontoparallel plane is almost always in the direction expected from the projection into the distal world of the relative motion of the images on the eye. It is hypothesized that this is the result of the perceptual underestimation of the depth between the objects. If a perceptual overestimation of the depth were produced, it was predicted that the apparent relative motion would be in a direction opposite to that expected from the projection of the retinal motions. This prediction was tested using the binocular disparity cue to produce perceptual overestimation of the slant (depth) of a luminous line. In this case, perceived slant was the indicator of perceived depth, and perceived rotation concomitant with the motion of the head was the indicator of perceived relative motion. The results support the prediction and also provide some support for a theoretically derived equation specifying the relation between these two perceptual variables.
\end{abstract}

Apparent concomitant motion is the apparent motion of objects that occurs concomitant with a motion of the head. It has been shown that this apparent motion can occur even though the objects physically do not move or do not move in this manner. This has been demonstrated for situations in which only one object is visible (Gogel, 1976, 1977; Hay \& Sawyer, 1969; Wallach, Yablick, \& Smith, 1972) and also for situations in which a number of objects are present simultaneously (Gogel, 1979; Gogel \& Tietz, 1977). The latter case can be illustrated by monocularly viewing two fingers held at different distances while moving the head from side to side. An apparent relative motion between the two physically stationary fingers will be observed, with the near finger appearing to move, relative to the far finger, in a direction opposite to that of the head motion. There are two explanations of this apparent relative motion. One notes that the direction of this apparent motion is consistent with the relative motions of the images of the fingers on the retina. It is as though the observer directly senses the retinal motion, which then determines the perceived motion. The second asserts that retinal motion in conjunction with perceived distance determines perceived motion (Gogel, 1976, 1977, 1979; Gogel \& Tietz, 1974, 1977). This latter explanation, if correct, has two important implications. The first is that the many instances of apparent motion obtained when stationary objects are viewed with a moving head in visually rich environments reflect the presence of errors in perceived distance. It is concluded that, although the three-

This research was supported by Research Grant BNS 77-16620 from the National Science Foundation. The author wishes to thank Jerome D. Tietz for his assistance in constructing the apparatus and gathering and analyzing the data. dimensional world seems (subjectively) to be well structured in distance, the perception of depth or distance is often in error and that this occurs despite the presence of a variety of distance cues. The second implication is that the perception of motion cannot be explained by the direct sensing of retinal motion. Instead, it is asserted that perceived distance always is an essential component in the perception of motion. The present study documents the inability of retinal motion by itself to predict apparent concomitant motion. In particular, the hypothesis is tested that, consistent with the explanation in terms of perceived distance, apparent concomitant motion can occur in a direction opposite to that expected solely from retinal motion.

The relation between retinal motion, errors in perceived distance, and apparent concomitant motion for the case of viewing two points at different physical distances is illustrated in Figure 1. In this figure, the observer, while moving the head laterally between Positions 1 and 2 , views two physically stationary points of light in an otherwise dark visual field, with point $f$ physically more distant than point $n$. The apparent positions of the points are indicated by the open circles and the prime notation; the physical positions are indicated by the filled circles and the notation without primes. The relative motions of the points on the eye are determined by the physical distances of the points from the observer. Since the physical distances are the same in Figures $1 \mathrm{~A}$ and 1B, motions of the retinal images of the points are identical in Figures $1 \mathrm{~A}$ and $1 \mathrm{~B}$. Retinally, the image of $\mathrm{f}$ is to the left of the image of $n$ when observed from Position 1 and to its right when observed from Position 2 . If the relative retinal motions were perceived correctly and projected into the visual field, point $f$ would be perceived as moving in the direction of the 


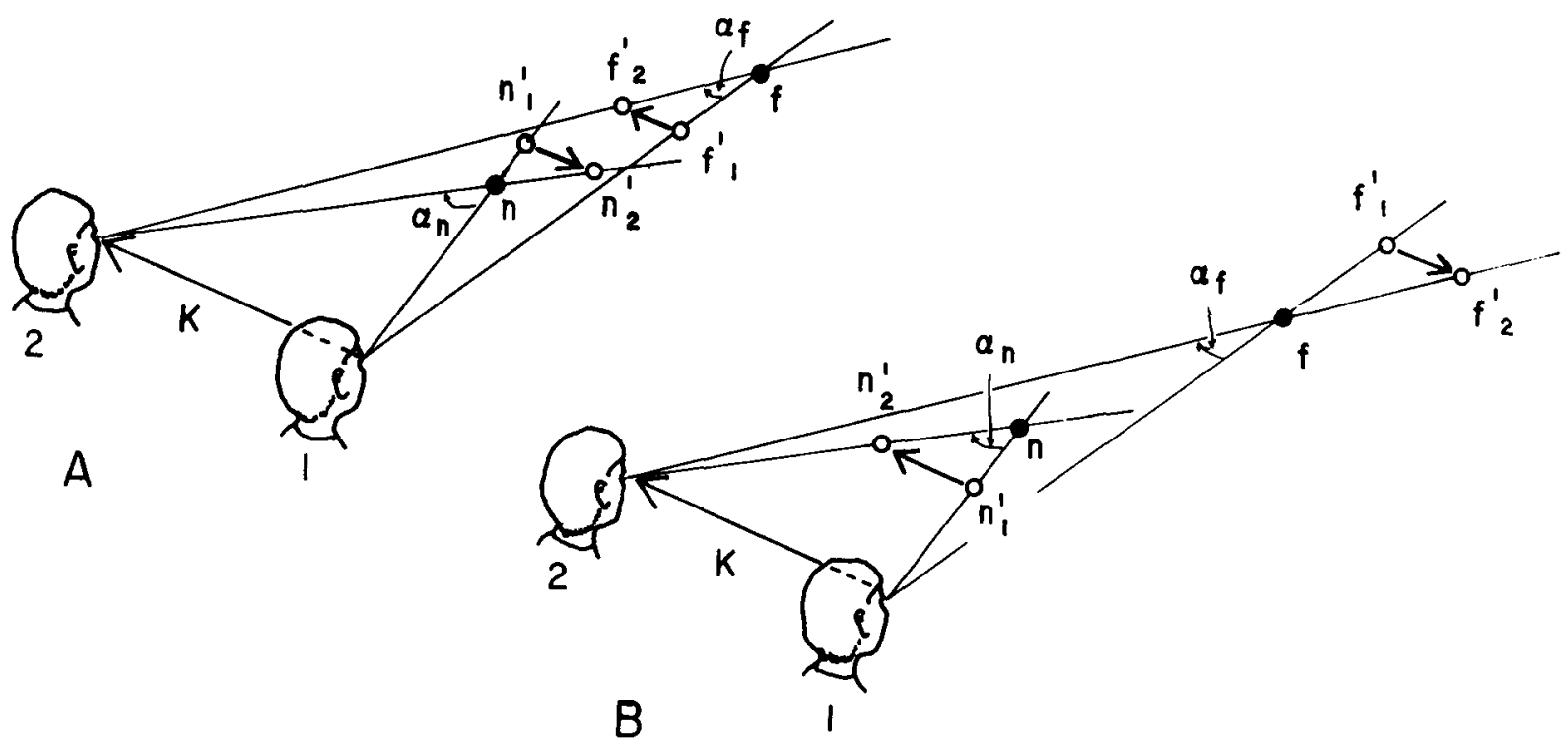

Figure 1. Apparent relative motion, concomitant with a lateral motion of the head, for two points at different physical distances ( $n$ and $f$ ). In $A$, the perceived depth between the two points is less than the physical depth and the apparent relative motion is consistent with that expected solely from the relative motion on the retina. In $B$, the perceived depth between the two points is greater than the physical depth and the apparent relative motion is opposite to that expected solely from the relative motion on the retina.

head motion (relative to point $\mathrm{n}$ ) and point $\mathrm{n}$ would be perceived as moving opposite to the direction of the head motion (relative to point $\mathrm{f}$ ).

The effect of apparent relative distance on apparent relative motion concomitant with a motion of the head can be illustrated by considering three instances of the apparent distances of points $n$ and $f$. Suppose, in one instance, that the distances of the points are correctly perceived. In this case, as the head is moved, despite the relative movements on the eye, the two points will be perceived as stationary. The observer will perceive that he is viewing two stationary points from different positions. As a second instance, consider the situation in Figure $1 \mathrm{~A}$ in which the depth between points $n$ and $f$ is perceptually underestimated; that is, point $n$ is perceived to be more distant, and point $f$ is perceived to be less distant, than its physical distance. In this case, the points will appear to move opposite to each other in the directions indicated by the arrows. The perceived motions are consistent with those expected solely from the relative motions on the eye; that is, they possibly can be explained by the direct perception of retinal motion. Finally, Figure 1B illustrates the situation in which the depth between points $n$ and $f$ is perceptually overestimated; that is, point $\mathbf{n}$ appears nearer, and point $f$ appears more distant, than its physical distance. In Figure 1B, point $n$ appears to move in the same direction as the head and point $f$ appears to move opposite to the head motion. The apparent relative motion of the points is opposite to that expected solely from the relative motion on the eye.
The relative motion of the two points on the eye expressed in radians is proportional to the difference between the angles $\alpha_{\mathrm{n}}$ and $\alpha_{\mathrm{f}}$ subtended by the head motion $\mathrm{K}$ at points $\mathrm{n}$ and $\mathrm{f}$ located at the physical distances $D_{n}$ and $D_{f}$ from the observer; that is, the equation for relative retinal motion is

$$
\alpha_{\mathrm{n}}-\alpha_{\mathrm{f}}=\mathrm{K} / \mathrm{D}_{\mathrm{n}}-\mathrm{K} / \mathrm{D}_{\mathrm{f}} .
$$

For the condition shown in Figure 1, in which both $\mathrm{n}$ and $\mathrm{f}$ are physically stationary as the head is moved, the difference in the perceived concomitant motion $\mathrm{W}_{\mathrm{n}}^{\prime}$ of point $\mathrm{n}$ and $\mathrm{W}_{\mathrm{f}}^{\prime}$ of point $\mathrm{f}$, taking apparent distance into account (see Gogel \& Tietz, 1974), can be expressed as

$$
W_{n}^{\prime}-W_{f}^{\prime}=\left(K / D_{f}\right) D_{f}^{\prime}-\left(K / D_{n}\right) D_{n}^{\prime}=\alpha_{f} D_{f}^{\prime}-\alpha_{n} D_{n}^{\prime} .
$$

According to Equation 2, the perception of relative motion is a function of both the retinal motions $(\alpha)$ and the perceived distances $\left(D^{\prime}\right)$ of the points from the observer. It follows that the final perception need not resemble the retinal motion. Equation 2 as readily predicts apparent concomitant motions inconsistent with the direction expected solely from retinal motions as it does those consistent with it.

The perceptual underestimation of depth illustrated in Figure 1A occurs frequently, whereas the perceptual overestimation illustrated in Figure 1B, to my knowledge, has not been demonstrated. The ubiquitous occurrence of the perceptual underestimation of depth can be attributed to the equidistance tendency, the tendency for stimuli to appear to be at the same 
distance (equidistant) in the absence of effective cues of depth. If some depth information is available, as is usually the case, the perceived depth within the configuration (as indicated by Figure 1A) will be decreased, but not eliminated. This applies to the perceived depth between parts of objects as well as between different objects. For example, a line or surface slanted in depth will appear less slanted as a consequence of the equidistance tendency. Also, there is evidence that the perceived distance of a nonfixated object is modified more by this tendency than is that of a fixated object, with the former displaced in apparent depth toward the latter (Gogel \& Tietz, 1977; Wist \& Summons, 1976).

To achieve the perceptual overestimation of depth required by Figure 1B, a depth cue must be introduced that is able to reverse the underestimation of depth normally resulting from the equidistance tendency. In the present study, the depth cue used for this purpose is binocular disparity. A luminous straight line, rather than the two points of Figure 1, was used to minimize the effect of diplopic images. This line formed an angle with the vertical plane and is illustrated by the line bt in Figure 2. As the principles of Figure 1 suggest, if the apparent slant of the line bt is different from its physical slant, the line will appear to rotate through an angle $\left(\beta^{\prime}\right)$ as the head is moved laterally between Positions 1 and 2 . Figure $2 \mathrm{~A}$ illustrates the situation in which the perceived slant is opposite in direction to the physical slant. This is an extension of the perceptual underestimation of depth shown in Figure 1A, and the direction of $\beta^{\prime}$ will be consistent with that expected solely from the direction of rotation of the image of the line on the eye. Figure 2B illustrates the situation in which the perceived slant is in the same direction as, but greater than, the physical slant. In this case, consistent with the principles illustrated by Figure 1B, the perceived direction of rotation of the line will be opposite to that expected solely from the direction of rotation of the image of the line on the retina. A case analogous to that of Figure 2B will occur if the line tb is slanted with the top ( $\mathrm{t}$ ) less distant than the bottom (b) and if the perceived slant in this direction is greater than the physical slant. In this case, also, the direction of the perceived rotation of the line will be opposite to that expected solely from the direction of rotation of its image on the retina. In the present study, the physical slant $(\phi)$, and thus the physical depth between the ends of the line tb, was varied. The perceived slant $\left(\phi^{\prime}\right)$, and thus the perceived rotation $\left(\beta^{\prime}\right)$, of the line tb between the apparent positions $\mathrm{t}_{1}^{\prime} \mathrm{b}_{1}^{\prime}$ and $t_{2}^{\prime} b_{2}^{\prime}$ also was varied for a constant value of $\phi$, by changing the stereoscopic slant of the line using polarizing filters. The relation between perceived slant $\left(\phi^{\prime}\right)$ and perceived rotation $\left(\beta^{\prime}\right)$, for a given value of physical slant $\phi$, is

$$
\tan \beta^{\prime}=\frac{K \sin \left(\phi^{\prime}-\phi\right)}{\mathrm{D} \cos (\phi+\lambda)},
$$

where $\lambda$ is the visual angle formed between the center of the line (at the level of the observer's eyes) and either the upper or lower end of the line physically at the distance $D$ from the observer, and $K$ is the lateral extent of the motion of the head. The value of $\lambda$ is positive or negative, depending upon whether it is defined by the upper or lower end of the line. The values of $\phi^{\prime}$ and $\phi$ are positive when the line appears or is, respectively, slanted with the top back and are negative when the line appears or is slanted with the top forward. The direction of the apparent rotation will be consistent with that expected solely from the retinal rotation either when the perceived slant is in the same direction as the physical slant but is less slanted than the physical slant or when the perceived slant is opposite to the physical slant. Only if the perceived slant is both in the same direction as, and is greater than, the physical slant will the direction of the perceived rotation predicted from Equation 3 be
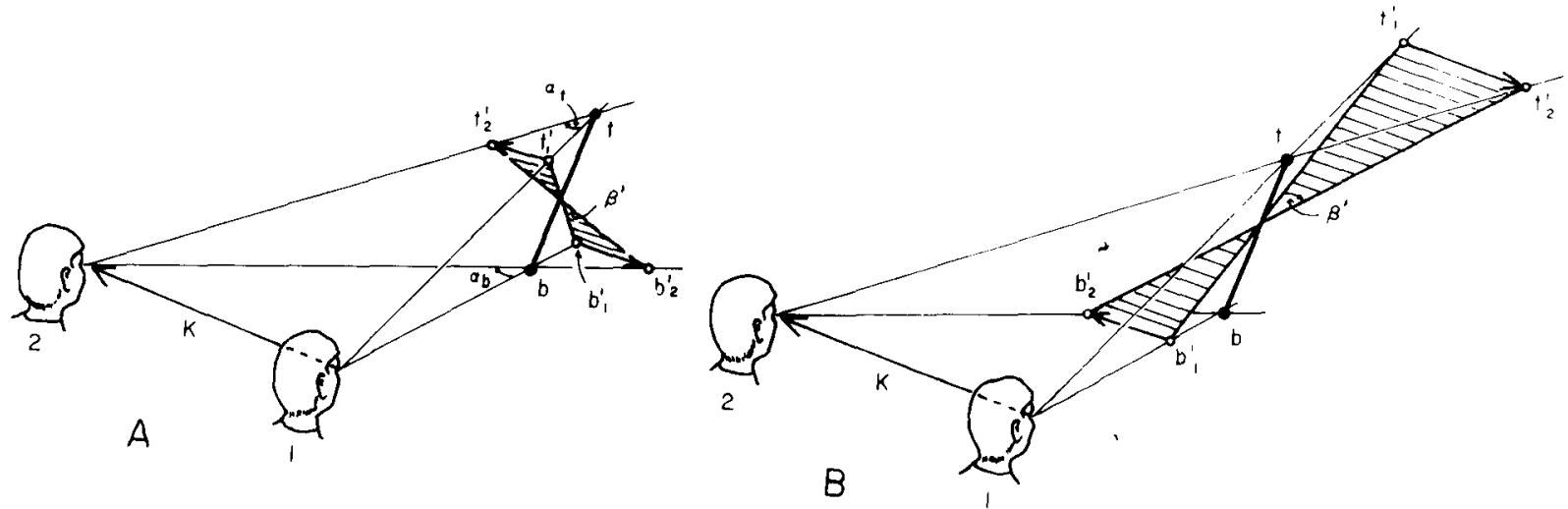

Figure 2. Apparent rotation concomitant with a lateral motion of the head of a line (tb) slanted in depth, with t physically more distant than $b$. In $A$, the perceived slant is opposite to the physical slant and the apparent rotation $\left(\beta^{\prime}\right)$ of the line is consistent with that expected solely from the rotation on the retina. In $B$, the perceived slant is in the same direction as the physical slant but greater in magnitude and the apparent rotation $\left(\beta^{\prime}\right)$ of the line is opposite to that expected solely from the rotation on the retina. 
opposite to that expected solely from the retinal rotation. It is this prediction that was to be tested. In the study, $\phi, \lambda, \mathrm{K}$, and $\mathrm{D}$ were given by the experimental conditions and the problem was to determine whether the magnitude and direction of $\beta^{\prime}$ could be predicted from $\phi^{\prime}$.

\section{EXPERIMENT 1}

\section{Observers \\ Method}

The observers were six graduate students (four men and two women) in the Psychology Department who were paid for their participation. All had an acuity of $20 / 20$ in each eye near and far and a stereoscopic acuity of at least $18.04 \mathrm{sec}$ of arc as measured in the Keystone orthoscope. Several had participated in a previous experiment involving apparent concomitant motion, but none knew the purpose of the present experiment.

\section{Apparatus}

The apparatus is illustrated in Figure 3. The observer is shown facing the portion of the apparatus that generated the line stimulus, with the portion for indicating the perceived slant and perceived rotation of the stimulus line located to the right of the observer. The observation position containing the head- and chinrest and the response portion of the apparatus was in a lightproof booth. The head- and chinrest was movable on rollers through a left-to-right distance of $15.2 \mathrm{~cm}$ with hard cushioned stops at each end of the travel. A click from a metronome was presented through a speaker every $1.5 \mathrm{sec}$ to pace the rate at which the observer arrived at the stops.

The line stimulus. The stimuli producing the perceived stimulus line were viewed through a nonrestrictive aperture that could be occluded by a shutter operated from outside the observation booth. The stimuli were two straight luminous lines, $21.5 \mathrm{~cm}$ long and $.8 \mathrm{~mm}$ wide, produced by masking electroluminescent "tape light" strips. As shown in Figure 3, one line was viewed by reflection from a 45 -deg mirror and the other was seen directly through the mirror. Polarizing filters, located in front of the stimuli and also in the goggles worn by the observer, resulted in the reflected line's being seen by the right eye only and the other line's being seen by the left eye only (see Figure 3 ). The midpoint

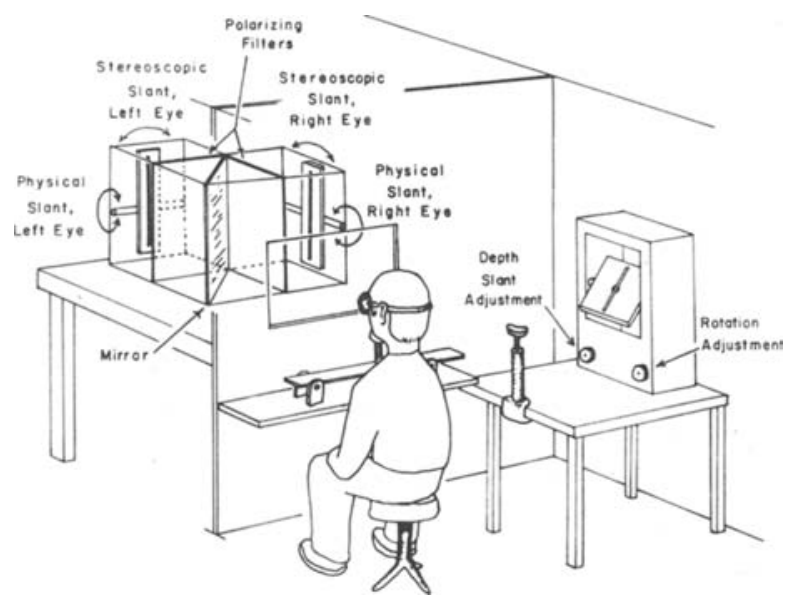

Figure 3. Schematic drawing of the apparatus for generating the stimulus line. The stimulus line is shown in the figure directly in front of the observer, and the response apparatus used for indicating the perceived slant $\left(\phi^{\prime}\right)$ and the perceived rotation $\left(\beta^{\prime}\right)$ of the line stimulus is shown as located to the observer's right. of each line was at eye level and optically at $100 \mathrm{~cm}$ from the observer's eyes. The lines had a luminance of $1.64 \mathrm{~cd} / \mathrm{m}^{2}$ as viewed through the polarizing filters. When the line stimuli were being viewed, the observation booth was in darkness, and when binocularly fused, the luminous lines were seen as a single line in an otherwise totally dark visual field.

As shown in Figure 3, the experimenter could rotate the lines together in depth around their centers to vary the physical depth slant $(\phi)$ of the binocularly viewed line. Each line also could be rotated $(\theta)$ optically around its center in opposite directions in the plane of its physical slant to vary the stereoscopic slant of the binocularly fused line and thus to vary in opposite directions the convergence angle to the two ends of the line. The total stereoscopic slant $(\Gamma)$ of the stimulus line from the vertical was a function of both $\phi$ and $\theta$, whereas the physical depth slant of the line was specified by $\phi$ alone. The magnitudes of $\phi$ and $\theta$ were adjusted by the experimenter to the required values from the back of the display apparatus. Three physical depth slants of the line around its center were used-20 deg from the vertical with the top near $(\phi=-20 \mathrm{deg})$, vertical $(\phi=0 \mathrm{deg})$, and $20 \mathrm{deg}$ from the vertical with the top far $(\phi=+20 \mathrm{deg})$. Five stereoscopic values, $\theta=-9.0,-4.5,0.0,+4.5$, and $+9.0 \mathrm{deg}$, were used at each of the three physical slants to produce a stereoscopic displacement of the line with respect to its physical plane of slant. A minus $\theta$ increased, and a plus $\theta$ decreased, the convergence to the top of the line relative to the bottom. When $\phi$ and $\theta$ had the same sign, they both contributed to perceiving a depth slant in the same direction from the vertical; when opposite in sign, they were opposed in determining the resulting stereoscopic slant. Retinal rotation was determined solely by the physical slant $(\phi)$ and was unaffected by the stereoscopic slant added by $\theta$. Both $\phi$ and $\theta$, however, by contributing to $r$, the combined (total) stereoscopic slant, were expected to modify perceived slant.

The 15 stereoscopic slants $(\Gamma)$ resulting from the combined effect of $\theta$ and $\phi$ are shown in three of the columns of Table 1. Because of the equidistance tendency, it was not expected that the perceived slant $\left(\phi^{\prime}\right)$ of the line would be as large as $\Gamma$. Only when $\Gamma$ had the same sign and was larger in absolute magnitude than the physical slant was it at all likely that a perceived rotation would occur that was opposite in direction to that expected solely from the rotation on the eye.

The response apparatus. The observer indicated both the plane of apparent slant of the stimulus line and its apparent rotation concomitant with head motion using the apparatus shown on the right in Figure 3. This apparatus consisted of a stationary headrest, a square blue plate $(16.7 \mathrm{~cm}$ on a side) that could be varied in physical slant in depth by turning the knob at the left of the device, and a thin white metal rod $(15.3 \mathrm{~cm}$ long with a $1.3 \times 3.0 \mathrm{~mm}$ cross section) that could be rotated around its midpoint in the plane of the blue plate by turning the knob at the right. A potentiometer was attached to the axis of rotation of the plate and another to the axis of rotation on the rod. Varying the slant of the plate or the orientation (rotation) of the rod changed the resistance of a potentiometer, which caused a proportional change in voltage on a digital voltmeter, which could be converted to an angular measure accurate to $\pm 1 \mathrm{~min}$ of arc. A switch at the experimenter's position determined whether the voltmeter indicated the adjustment of apparent slant in depth $\left(\phi^{\prime}\right)$ or apparent rotation $\left(\beta^{\prime}\right)$. The observation for the response adjustments always was binocular, with the polarized goggles removed. The observer and experimenter communicated by microphones and speakers.

\section{Procedure}

First, with the observation shutter closed, the observer was given practice moving his head in the head- and chinrest from one side to the other in time with the metronome. The terms "apparent slant" and "apparent rotation," and the kinds of adjustment responses, were described using the response apparatus as a model. In making a response, the observer first oriented the plate on which the response rod was mounted to the depth slant perceived 
in the line stimulus on the immediately preceding experimental trial. The observer then indicated the angle through which the line had appeared to rotate in the plane of this slant by adjusting the response rod to one extreme (limit) of this angle and then to the other, pausing so that the experimenter could record each extreme. The observer repeated the rotation adjustments several times before the extreme rotational positions were recorded.

The general procedure was as follows. While wearing the goggles, and with his head in the head- and chinrest, the observer moved his head as far to the right as possible. The observation shutter was opened and the observer indicated whether the line appeared slanted in depth and, if so, its direction of slant. Following this, the observer moved his head once from right to left and reported the direction (clockwise or counterclockwise) of the apparent rotation of the line (if any occurred). The report for the right-to-left motion only of the head (not the converse) enabled the experimenter to determine whether the perceived rotation was in the direction of, or opposite to, that expected solely from the retinal rotation. Following this, the observer was asked to note the apparent slant and apparent magnitude of rotation while moving his head repetitively right and left and while moving his gaze along the stimulus line so as to minimize diplopic images. Since apparent slant and apparent rotation always occurred simultaneously in both images, however, slant and rotation judgments could be made even if double images of the line were observed. Following this, the shutter was closed, the booth light was turned on, the goggles were removed, and the observer turned to the response apparatus to indicate (using binocular observation) the slant and rotation perceived in the stimulus line. The observer initially received two practice trials with the stimulus line physically vertical $(\phi=0 \mathrm{deg})$ but stereoscopically at one of the extreme values $(\Gamma= \pm 65.5 \mathrm{deg})$. As expected, all observers perceived an apparent rotation of the luminous line under these conditions, as indicated by the adjustments on the response apparatus.

The 15 experimental conditions ( 15 values of $\Gamma$ ) were presented twice to each observer. All values of $\theta$ were presented at the same $\phi$ before a different value of $\phi$ occurred. The order of presentation of the different values of $\phi$ was counterbalanced between observers, with $\theta$ presented in a partially random, partially counterbalanced order that was different for each observer.

\section{Results}

The average perceived slants ( $\left.\phi^{\prime}\right)$ in depth from the vertical and the average perceived rotations $\left(\beta^{\prime}\right)$ in the plane of the perceived slant of the line stimulus for Experiment 1 are shown in the upper portion of Table 1. A positive perceived slant means that the top of the line was perceived to be more distant than the bottom. A negative perceived slant means the converse. The effect of the equidistance tendency is evident in Table 1 in that the perceived slants are less than those expected from the combined stereoscopic slants (Г). A positive perceived rotation means that the stimulus line was perceived to rotate clockwise as the head was moved left; a negative perceived rotation means that the stimulus line was perceived to rotate counterclockwise as the head was moved left. If the direction of retinal rotation determined the direction of perceived rotation independently of perceived slant, the perceived rotation as the head moved left would be always negative for $\phi=+20 \mathrm{deg}$, zero for $\phi=0 \mathrm{deg}$, and positive for $\phi=-20 \mathrm{deg}$.

According to the discussion of Figures $1 \mathrm{~B}$ and $2 \mathrm{~B}$, the direction of the perceived rotation $\left(\beta^{\prime}\right)$ of the line would be opposite to that expected solely from the retinal rotation if, and only if, the perceived depth slant of the line, in the same direction as the physical slant, was greater than its physical slant. The perceived slant data, identified by underlining in Table 1 , satisfy this condition. For $\theta$ values of +4.5 and $+9.0 \mathrm{deg}$ in Experiment 1, the line physically slanted at $\phi=+20 \mathrm{deg}$ (top back) was perceived, on the average, to be slanted (top back) at +36.5 and $+53.7 \mathrm{deg}$, respectively. Under these conditions, as pre-

Table 1

Means and Standard Deviations of Perceived Slant $\left(\phi^{\prime}\right)$ From the Vertical and Perceived Rotation $\left(\beta^{\prime}\right)$ in Degrees in the Plane of Apparent Slant as a Function of the Combined Stereoscopic Slant ( $\Gamma$ ) Produced by Three Physical Slants ( $\phi)$ From the Vertical Together With Five Stereoscopic Displacements $(\theta)$

\begin{tabular}{|c|c|c|c|c|c|c|c|c|c|c|c|c|c|c|c|}
\hline \multirow[b]{3}{*}{$\theta$} & \multirow[b]{3}{*}{$\Gamma$} & \multicolumn{4}{|c|}{ Top Near $(\phi=-20 \mathrm{deg})$} & \multicolumn{5}{|c|}{ Vertical $(\phi=0 \mathrm{deg})$} & \multicolumn{5}{|c|}{ Top Far $(\phi=+20 \mathrm{deg})$} \\
\hline & & \multicolumn{2}{|c|}{$\phi^{\prime}$} & \multicolumn{2}{|c|}{$\beta^{\prime}$} & \multirow[b]{2}{*}{$\Gamma$} & \multicolumn{2}{|c|}{$\phi^{\prime}$} & \multicolumn{2}{|c|}{$\beta^{\prime}$} & \multirow[b]{2}{*}{ r } & \multicolumn{2}{|c|}{$\phi^{\prime}$} & \multicolumn{2}{|l|}{$\beta^{\prime}$} \\
\hline & & Mean & $\mathrm{SD}$ & Mean & $\mathrm{SD}$ & & Mean & $\mathrm{SD}$ & Mean & SD & & Mean & $\mathrm{SD}$ & Mean & SD \\
\hline \multicolumn{16}{|c|}{ Experiment 1} \\
\hline-9.0 & -69.7 & -34.9 & 17.8 & -.5 & 4.1 & -65.5 & -26.4 & 17.3 & -5.5 & 8.2 & -61.1 & -25.5 & 27.2 & -12.1 & 4.6 \\
\hline 4.5 & -56.1 & -21.3 & 18.9 & +.2 & 3 & -47.6 & -14.0 & 17.3 & -5.4 & 8.0 & -37.4 & -9.1 & 20.2 & -13.0 & 7 \\
\hline .0 & -18.5 & -1.0 & 1.8 & +7.5 & 6.8 & .0 & +2.5 & 4.5 & +1.0 & 1.7 & +18.5 & +8.4 & 7.2 & -2.6 & 2 \\
\hline 4.5 & +37.4 & +21.3 & 8.4 & +16.4 & 7.4 & +47.6 & +27.4 & 10.1 & +14.3 & 11.0 & +56.1 & +36.5 & 6.4 & +7.7 & 6. \\
\hline 9 & +61.1 & +45.3 & 8.4 & +19.9 & 8.0 & +65.5 & +42.6 & 13.1 & +17.2 & 7.5 & +69.7 & +53.7 & 6.2 & +15.3 & 7.8 \\
\hline \multicolumn{16}{|c|}{ Experiment 2} \\
\hline-9.0 & -69.7 & -34.2 & 20.5 & -.5 & 3.3 & -65.5 & -27.4 & 18.0 & -7.2 & 6.5 & -61.1 & -28.0 & 18.2 & -11.8 & 7 \\
\hline-4.5 & -56.1 & -20.1 & 14.2 & & 4.6 & -47.6 & -7.3 & 14.6 & -3.8 & 4.4 & -37.4 & -9.8 & 15.8 & -11.6 & \\
\hline .0 & -18.5 & +.6 & .9 & +4.8 & 4. & .0 & +1.4 & 1.6 & & .4 & +18.5 & +6.1 & 9.3 & -2.3 & 3. \\
\hline+4.5 & +37.4 & +19.0 & 8.1 & +12.1 & 5 . & +47.6 & +23.6 & 7.3 & +8.9 & 6.9 & +56.1 & +34.4 & 10.2 & +2.0 & 3.3 \\
\hline+9.0 & +61.1 & +42.7 & 7.8 & +9.8 & 7.1 & +65.5 & +38.9 & 10.7 & +7.8 & 9.4 & +69.7 & +49.9 & 13.7 & +6.8 & 5.7 \\
\hline
\end{tabular}

Note $-\Gamma$ is calculated using the average interpupillary distance $(6.3 \mathrm{~cm})$ of the six observers. For both $\phi$ and $\phi '$, plus values indicate a top far slant; minus values indicate a top near slan. Perceived slant in the same direction as phvsical slant exceeds physical slant appreciably only for underlined values. Plus and minus values of $\beta^{\prime}$ indicate a ciockwise and counterclockwise apparent rotation. respectively, as the head is moved to the left. 
dicted by Equation 3, the perceived rotation should be opposite in direction to the counterclockwise perception of rotation expected from the rotation on the eye when moving the head from Position 1 to Position 2 (right to left) and, as identified by the values of $\beta^{\prime}$ of +7.7 and $+15.3 \mathrm{deg}$, this was indeed the case. The +7.7- and +15.3-deg values of $\beta^{\prime}$ were different from zero $\left(\mathrm{t}_{5}=3.0, \mathrm{p}<.05\right.$, and $4.8, \mathrm{p}<.01$, respectively, with a one-tailed test). With the top physically near $(\phi=-20 \mathrm{deg})$, a slant appreciably greater than $-20 \mathrm{deg}$ was perceived, on the average, only when a value of $\theta$ of $-9.0 \mathrm{deg}$ was used. The perceived rotation in this case should have been negative to be opposite to that expected from the retinal rotation and, although this occurred on the average, it was almost zero $\left(\beta^{\prime}=-.5 \mathrm{deg}\right)$. Thus, clear evidence for a perceived rotation opposite in direction to that expected solely from the direction of rotation on the eye is present, but only for the case in which the perceived slant was greater than the physical slant, with the top more distant both physically and perceptually. It is pertinent, however, that the average perceived rotation predicted from the average perceived slant using Equation 3 was +2.6 and $+5.1 \mathrm{deg}$ for $\theta=+4.5$ and $+9.0 \mathrm{deg}$, respectively, for the top far condition $(\phi=+20 \mathrm{deg})$, as compared with the predicted value of $-2.4 \mathrm{deg}$ for $\theta=-9.0 \mathrm{deg}$ and $\phi=-20 \mathrm{deg}$. Thus, for the values of perceived slant obtained, the top far condition was more likely to demonstrate the inability of the direction of retinal rotation alone to specify the direction of perceived rotation.

The theoretical relation between $\phi^{\prime}$ and $\beta^{\prime}$ for values of $\phi^{\prime}$ between $\pm 90 \mathrm{deg}$, predicted from Equation 3 , for the physical values of $\phi, \Gamma, D$, and $K$ used in the study, is shown by the three curved dashed lines that, together, form the banana-shaped figure of Figure 4. If, contrary to Equation 3, the perception of rotation of the stimulus line were determined solely by the retinal rotation, each of the theoretical curves would be reduced to a horizontal line with an ordinate value specified by the intersection of the curved and vertical dashed lines. Thus, for $\phi=-20$, 0 , and $+20 \mathrm{deg}$, the perceived rotation predicted solely from the retinal rotation is $+3.1,0$, and -3.1 $\mathrm{deg}$, respectively, for all values of perceived slant.

The relations obtained in Experiment 1 between the averages of perceived slant and perceived rotation indicated in Table 1 are shown in Figure 4 by the solid lines. Since each of the physical slants of the stimulus line produced a nonzero slope, it is clear that perceived rotation was not determined exclusively by retinal rotation; rather, it varied systematically with perceived slant. The circled points in Figure 4 are results from the conditions in which perceived slant was in the same direction as, but appreciably larger than, the physical slant. These are the conditions in which it was expected (and obtained

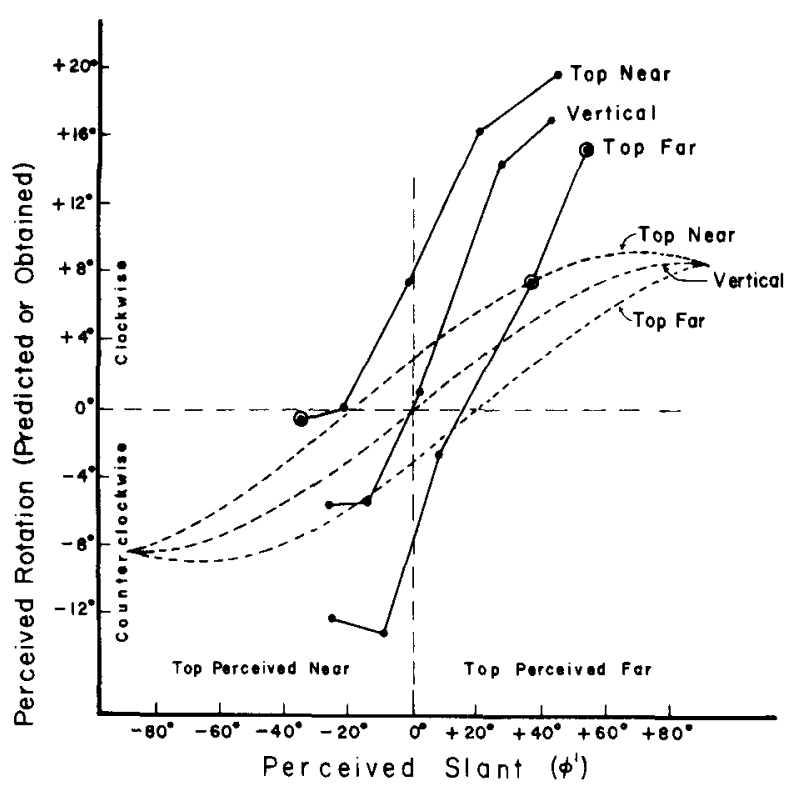

Figure 4. The theoretical (curved dashed lines) and obtained (curved solid lines) relations between perceived slant $\left(\phi^{\prime}\right)$ and perceived rotation $\left(\beta^{\prime}\right)$ from Experiment 1.

in the case of $\phi=+20 \mathrm{deg}$ ) that the perceived rotation would be opposite to that predicted solely from the retinal rotation.

It also should be noted that, although no retinal rotation of the stimulus line occurred concomitant with head motion when the stimulus line was physically vertical, substantial amounts of perceived rotation in the directions predicted from Equation 3 were obtained when the line was perceived as slanted in depth. This result is consistent with that obtained by Gogel and Tietz (1974) using an illusory slant produced by the perspective cue. It is clear that the perceived rotation of the line under any of the conditions of physical slant cannot be explained as a direct perception of retinal rotation.

Figures 4 and 5 permit the ready comparison of the outcomes predicted from Equation 3 and those obtained in the experiment. Although the shapes of the obtained data curves in Figure 4 have some similarity to the shapes of the theoretical curves resulting from Equation 3, the absolute values of perceived rotation measured in the experiment often are considerably larger for a given perceived slant than predicted. Figure 5 shows the best fitting straight line obtained by the method of least squares, relating the average measured perception of rotation ( $\beta^{\prime}$ obtained) and the perception of rotation expected from Equation 3 ( $\beta^{\prime}$ predicted). The predicted $\beta^{\prime}$ was calculated by substituting, in Equation 3, the average perceived slants $\left(\phi^{\prime}\right)$ of the stimulus line obtained for the different combinations of physical variables. The Pearson product-moment correlation was +.98 . It follows that the magnitude and direction of the obtained apparent rotations were closely related to those pre- 


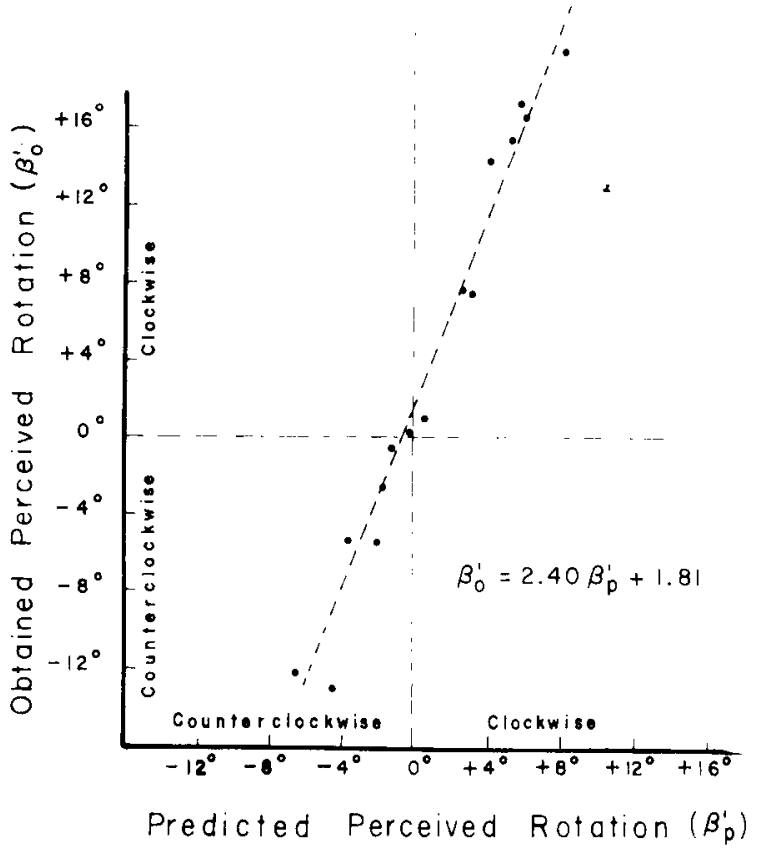

Figure 5. The relation between the perceived rotation $\left(\beta_{p}^{\prime}\right)$ predicted from Equation 3 using average values of perceived slant $\left(\phi^{\prime}\right)$ and the perceived rotation $\left(\beta_{0}^{\prime}\right)$ obtained directly from Experiment 1.

dicted, except that the obtained apparent rotations were 2.4 times as large as predicted. The purpose of Experiment 2 was to examine this discrepancy between the magnitude of the predicted and obtained results.

\section{EXPERIMENT 2}

\section{Method}

Perhaps the discrepancy between the data curves and the theoretical curves of Figure 4 is the result of systematic psychophysical errors in the measurement of $\phi^{\prime}$ or $\beta^{\prime}$. One factor possibly contributing to a measurement error in $\beta^{\prime}$ is that, particularly for the larger stereoscopic slants, the stimulus line appeared to be much longer than the response line as suggested by casual observation. A consequence of this is that, for a given perceived angle of rotation, the apparent motion of the ends of the stimulus line exceeded that of the ends of the response line. In Experiment 1, the observers had been asked simply to indicate (using the response line) the angle through which the stimulus line appeared to rotate as they moved their heads. In Experiment 2, the instructions made a distinction between the amount of perceived motion of the ends of the line and direct judgment of the angle of apparent rotation. This distinction was illustrated by means of a drawing, and the observer was instructed explicitly to avoid using the magnitude of the perceived motion of the ends of the line in indicating the rotation perceived in the stimulus.

\section{Observers}

The observers were the same as those used in Experiment $\mathbf{l}$.

\section{Apparatus and Procedure}

The apparatus was identical in Experiments 1 and 2. The only difference between the two experiments was the change in instructions for measuring the perceived rotation of the stimulus line.

\section{Results}

The average results from Experiment 2 are shown in the lower portion of Table 1 and in Figures 6 and 7. Perceived rotation opposite to that expected solely from the retinal rotation occurred for the "top far" condition, with $\theta$ of +4.5 and $+9.0 \mathrm{deg}$, as indicated by the +2 - and +7 -deg perceived rotations, although only the latter value was reliably different from zero $\left(\mathrm{t}_{\mathrm{s}}=2.9, \mathrm{p}<.05\right.$, for a one-tailed test $)$. The values predicted from Equation 3, using the average $\phi^{\prime}$ obtained from these conditions, were 2.3 and $4.6 \mathrm{deg}$, respectively. As in Experiment 1, the top near condition for $\theta=-9.0 \mathrm{deg}$ did not result in appreciable apparent rotation. The value predicted from Equation 3 for the average $\phi^{\prime}$ obtained from this condition, however, was only $-2.2 \mathrm{deg}$. The curves relating the average obtained $\phi^{\prime}$ and $\beta^{\prime}$ are more like the theoretical curves in Figure 6 than those in Figure 4. Comparing these two figures, it can be seen that this improvement is the result of a reduction in the obtained values of $\beta^{\prime}$ using the changed instructions, particularly in the conditions in which the top of the line was perceived to be more distant than the bottom. The slope of the line of best fit relating $\beta^{\prime}$ predicted and $\beta^{\prime}$ obtained was reduced from 2.4 (Figure 5) to 1.6 (Figure 7), with the correlation between the predicted and obtained $\beta^{\prime}$ remaining at a very high value $(r=+.96)$. Although this change can be interpreted as a reduction of the artifact of judging the angle of apparent rotation at least partly by the motion of the ends of the stimulus and response lines, this is not the only possible interpretation. Possibly a practice effect (since the same observers were used) contributed to these results. In any event, the perceived rotations measured in Experiment 2 more

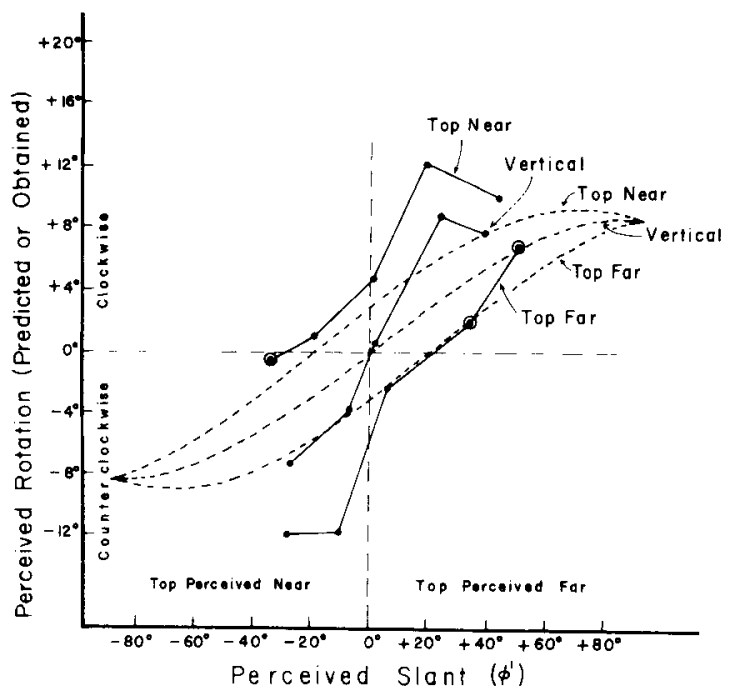

Figure 6. The theoretical (curved dashed lines) and obtained (curved solid lines) relations between perceived slant $\left(\phi^{\prime}\right)$ and perceived rotation $\left(\beta^{\prime}\right)$ from Experiment 2. 


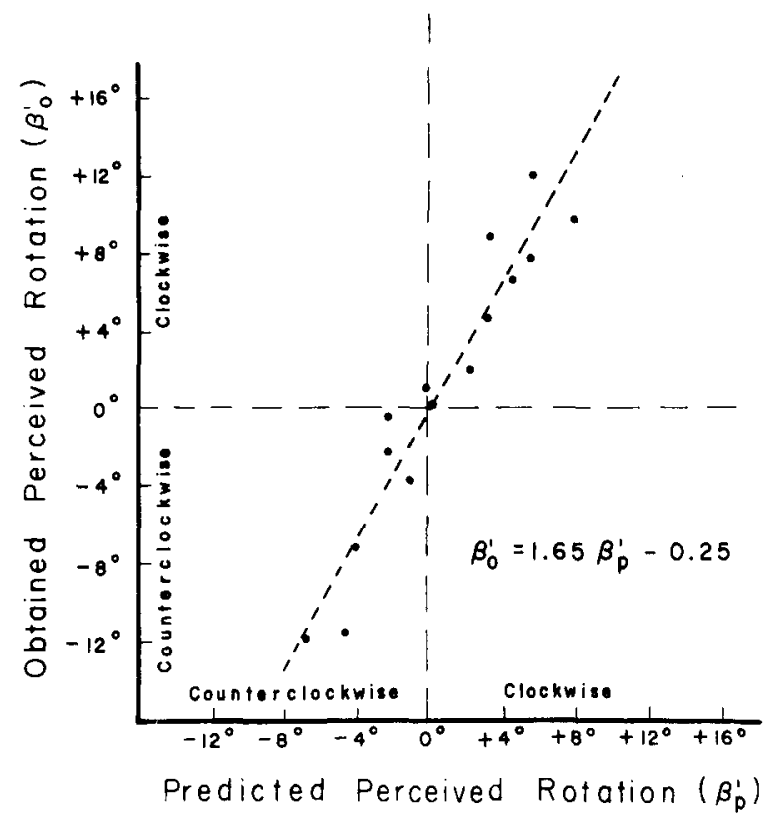

Figure 7. The relation between the perceived rotation $\left(\beta_{p}^{\prime}\right)$ predicted from Equation 3 using the average values of perceived slant $\left(\phi^{\prime}\right)$ and the perceived rotation $\left(\beta_{0}^{\prime}\right)$ obtained directly from Experiment 2.

nearly approximate the predicted values of perceived rotation than those obtained from Experiment 1. However, if Equation 3 is, indeed, correct, some additional, as yet unidentified, artifacts in the measurement of either $\beta^{\prime}$ or $\phi^{\prime}$ remain.

Experiment 2, like Experiment 1, provides clear evidence that, under the appropriate conditions, perceived rotation concomitant with a motion of the head can be in a direction opposite to that expected solely from retinal rotation. More generally, this study indicates that, under any conditions of observation, apparent concomitant rotation cannot be explained by retinal rotation in the absence of information regarding apparent slant.

\section{DISCUSSION}

\section{Errors in Perceived Distance}

The emphasis in the present study is on the prediction of errors in apparent concomitant rotation from errors in apparent slant. Conversely, errors in apparent rotation (or apparent relative motion) signify the presence of errors in apparent slant (or apparent depth). Indeed, as indicated by Equation 3, a physically stationary line can appear as stationary $\left(\beta^{\prime}=0\right)$ only when the perceived slant $\left(\phi^{\prime}\right)$ of the line is equal to its physical slant $(\phi)$, that is, only when there are no errors in perceived depth. The results of this study are consistent with those of previous studies (Gogel, 1979; Gogel \& Tietz, 1974, 1977) in which the apparent concomitant motion of physically stationary objects was shown to reflect the presence of errors in perceived distance or slant. Since such apparent motions, concomitant with the motion of the head, occur frequently even under multicue conditions of observation, the present study adds support to the conclusion that errors in perceived depth or distance are a common occurrence in normal visual fields. The direction of the errors is usually consistent with the operation of the equidistance tendency; that is, the apparent depth between the points or objects is less than the physical depth. It follows, from Equations 2 and 3, that, under these conditions, the direction of the apparent concomitant motion will be in the direction expected solely from the retinal motion. It is clear, however, that this result does not represent a direct response to the retinal motion, since, as the present study shows, perceived concomitant motion can occur with a stationary retinal image and can occur in a direction inconsistent with that expected solely from retinal motion.

Equations 2 and 3 are expected to hold regardless of the reason for the error in perceived depth or perceived slant. In the present study, errors in perceived slant were manipulated by the binocular disparity cue; Gogel and Tietz (1974) produced such errors by misleading perspective. It seems likely that the relation between $\phi^{\prime}$ and $\beta^{\prime}$ expressed by Equations 2 and 3 is independent of the manner in which $\phi^{\prime}$ is produced.

\section{Direct Perception of Retinal Motion}

A point of view opposed to that of the present study is expressed by the core-context hypothesis (Boring, 1952). According to this hypothesis, the contribution made by the retinal core stimulus (e.g., the retinal motion) to the perception of motion varies inversely with the amount of context (e.g., distance cues) available. Although the core-context hypothesis, like Equations 2 and 3, affirms the contribution of perceived distance to another perception (in these experiments, the perception of apparent concomitant motion), it differs in two respects from the point of view of this study. The core-context hypothesis suggests that the contribution of perceived distance to the perception becomes less as distance cues are reduced, and it predicts also that under the condition of total reduction in distance cues the perception will be determined only by the core stimulus. The corecontext hypothesis contains the assumption that, under reduced conditions and perhaps to some degree under all conditions, the observer has direct perceptual access to the retinal information. Equation 3 also specifies a condition under which the perception of rotation is proportional to the retinal core stimulus. This is the condition in which the stimulus line is perceived to be in a frontoparallel plane. For example, in the present study, only if the stimulus line is perceived as frontoparallel $\left(\phi^{\prime}=0\right)$ will its per- 
ceived rotation be equal in magnitude and direction to that expected solely from its retinal rotation. The theory underlying Equation 3, however, differs from the core-context hypothesis in that the perception of the orientation of the line (in this case, the perception of the line as vertical) is as necessary to the perception of rotation here as it is in any other condition. Indeed, if all distance cues were removed so that no information regarding perceived slant were available, the equidistance tendency would supply a perception of line slant, that is, a perception that the line is vertical. Again, however, this perceived orientation is as essential to the perception of the rotation of the line in this case as it is under any other condition. Unlike the core-context hypothesis, the point of view of the present study denies that perceived slant (or perceived distance) is less important for the perception of rotation (or for other perceptions) under some conditions than under others. According to the core-context hypothesis, in the absence of context the core is sufficient to determine the perception. According to the present study, without some perception of distance (whether veridical or in error), a perception of size, slant, or motion is impossible.

The difference between the core-context and the present point of view has experimental and theoretical consequences. One area in which this is evident is the study of constancy phenomena. Constancy phenomena are defined in terms of the differences between distal and proximal stimulation, with the degree of constancy specified by noting where the perception falls between these two alternative ways of defining stimuli. Zero constancy is said to occur when the perception resembles the proximal (core) stimulus, and perfect constancy is said to occur when perception is proportional to the distal stimulus. Perceptions intermediate between those expected from proximal and distal stimuli indicate some degree of underconstancy.

To understand the consequences of this description of constancy phenomena, consider its application to the study of the perceptual development in the infant. Suppose it is found that an infant exhibits zero constancy of size, shape, or motion in a particular situation. It can be concluded in this case that the infant is not utilizing distance cues if these are available. It cannot be concluded, however, that depth or distance was not perceived, or that the perception of size, shape, or motion was determined solely by the size, shape, or motion on the eye. If the basic requirements for perception in the infant are the same as in the adult, some perception of distance, determined perhaps by observer tendencies such as the equidistance tendency, must have been present. Thus, the problem is not only whether distance information is utilized, but also whether the perceptual sys- tem of the infant requires the kinds of interactions between perceptual variables found in the present study for the adult.

The core-context hypothesis and its implicit, but questionable, assumption of the direct perception of retinal stimulation continues to influence theory and research in spatial perception in a variety of ways. One additional example will be provided. It is noted often that the visual world appears to be stationary as the eyes are turned, a phenomenon that usually is attributed to the corrective influence of extraretinal information. Basically, such an interpretation supposes that, if the extraretinal signals were absent, the perception of position or motion would be determined exclusively by retinal position or by the change in retinal position. This, again, is an assumption of the direct perception of a retinal core stimulus and is not justified by the results of the present study. Extending the point of view of the present study, a more plausible explanation for this phenomenon is that the visual system always utilizes some value of extraretinal information, whether veridical or not, in order to arrive at a perception of object motion associated with eye rotation. The assumption that the spatial perception of motion, size, and perhaps other characteristics under any conditions results from direct access to the proximal stimulus is unnecessary and, from the present study, is incorrect.

\section{REFERENCES}

Boring, E. G. Visual perception as invariance. Psychological Review, 1952, 59, 141-148.

Gogel, W. C. An indirect method of measuring perceived distance from familiar size. Perception \& Psychophysics, 1976, 20, 4!9-429.

GogFL, W. C. An indirect measure of perceived distance from oculomotor cues. Perception \& Psychophysics, 1977, 21, 3-11.

Gogel, W. C. The common occurrence of errors of perceived distance. Perception \& Psychophysics, 1979, 25, 2-11.

Gogel., W. C., \& Newton, R. E. An apparatus for indirect measurement of perceived distance. Perceptual and Motor Skills, 1976, 43, 295-302.

GogEl, W. C., \& TIETz, J. D. The effect of perceived distance on perceived movement. Perception \& Psychophysics, 1974, 16, 70-78.

Gogel, W. C., \& TiEtz, J. D. Eye fixation and attention as modifiers of perceived distance. Perceptual and Motor Skills, $1977,45,343.362$.

Hay, J. C., \& SAWyer, S. Position constancy and binocular convergence. Perception \& Psychophysics, 1969, 5, 310-312.

Wallach, H., Yablick, G., \& Smith, A. Target distance and adaptation in distance perception in the constancy of visual direction. Perception \& Psychophysics, 1972, 12, 139-145.

Wist, E. R., \& Summons, E. Spatial and fixation conditions affecting the temporal course of change in perceived relative distance. Psvchological Research, 1976, 39, 99-112.

(Received for publication December 10, 1979; revision accepted May 20, 1980.) 